\title{
Snoezelen to Promote Improved Emotional Status in Stroke Caused by Defoliat Exposure in the Vietnam War: Case Study
}

\author{
Su-Kyoung Lee ${ }^{1}$, Sang-Yeol Lee ${ }^{2 *}$, Myoung-Kwon Kim ${ }^{3}$ \\ ${ }^{1}$ Department of Physical Therapy, Gimhae College, Gimhae-si, Republic of Korea; ${ }^{2}$ Department of Physical Therapy, Kyoungsung \\ University, Busan, Republic of Korea; ${ }^{3}$ Department of Physical Therapy, Youngsan University, Yansansi, Republic of Korea. \\ Email: *holeintwo@naver.com
}

Received October $22^{\text {nd }}, 2012$; revised November $9^{\text {th }}, 2012$; accepted December $9^{\text {th }}, 2012$

Copyright (C) 2013 Su-Kyoung Lee, et al. This is an open access article distributed under the Creative Commons Attribution License, which permits unrestricted use, distribution, and reproduction in any medium, provided the original work is properly cited.

\begin{abstract}
Objectives: The effects of snoezelen-based therapy on emotional status were investigated in Vietnam War veterans with brain injury suspected to be caused by defoliant exposure. Methods: A single subject A-B-A'-B' experimental design was used to determine the effects of snoezelen-based therapy. Results: A pegboard test to examine physical changes in a psychologically stable state, showed a decrease in measured time required from pegboard test. In the present study, which used an A-B-A'-B' research design, the first test showed that the snoezelen reduced tension-anxiety, anger-hostility and depression-dejection while the second test showed similar effects, except for fatigue-inertia and confusion-bewilderment. Conclusion: Snoezelen therapy had a positive effect on emotional stability and cognitive ability in a patient with emotional instability due to combat experience in the Vietnam War and with stroke caused by defoliant exposure.
\end{abstract}

Keywords: Defoliant; Snoezelen; Stroke

\section{Introduction}

Approximately 320,000 Korean soldiers fought in the Vietnam War (1955-1975) 5000 died and 310,000 survived and returned home. Many were exposed to defoliants used by the US military forces, which caused after effects such as skin disorders and cerebrovascular diseases that continue even now, 50 years after the war [1]. Brain injury due to cerebrovascular diseases has been reported as a suspected aftereffect symptom.

Most brain injury patients show rage and anger. The risk of these attacks is only $15 \%$ within one year of the injury, but this increases to $54 \%$ five years later, with accompanying emotional and physical disability [2]. Emotional instability associated with brain injuries, can be reduced by a number of methods, including the multisensory stimulation program, which is a sensory method. This method was, initially used with adults with learning disorders, but is currently used in connection with emotional status, including emotional disorder, allayment of

*Corresponding author. feelings excited by problem behavior, and stress management [3].

A multi-sensory stimulation program can use a snoezelen room, which provides positive stimulations and a stress-free environment by installation of different lighting, relaxing music, aromas, and tactual objects that stimulate, visual, auditory, tactual, and olfactory senses [4]. The various stimulations are provided randomly, regardless of the concentration and intellectual level of patients, so that they can freely experience sensory stimulations [4]. Although snoezelen was previously used as a type of leisure activity for to those having intellectual problems, its value is now recognized as an attractive therapeutic environment that has beneficial effects, based on reports from caregivers and treatment staff and from various experimental studies [5].

The various sensory stimulations provided by snoezelen have a direct effect in helping subjects to relax and to reduce stress in their lives [6]. Therapeutic effects of snoezelen have been reported in elderly dementia patients, in children with mental retardation and learning problems, 
and in chronic pain patients common effects are enhancement of emotion or mood states, such as reduced anxiety, decreased hostility, increased happy feelings, and sedative effects. Snoezelen was also reported to help in reducing psychological stress and to improve quality of life [7]. In a study conducted with children with severe brain injury, several snoezelen environments themselves showed an effect in brain recovery and reduced heart rate, muscular tension, and excitation, without the need for application of ROM and stretching exercises for relaxation [8]. The application of snoezelen was also reported to alleviate pain by providing a psychological sedative effect [7].

These previous studies that confirm the therapeutic effects of multi-sensory environments were conducted primarily with the elderly dementia patients with abnormal behavior and occasionally on patients with psychiatric disorders, including children with autism or emotional instability. These studies have tended to focus on stress alleviation in normal adults, whereas little attention has been directed towards effects on stroke patients with normal cognition. The study by Schofield et al. [7], conducted with chronic back pain patients, indicated that snoezelen had pain alleviating effects through its psychological sedative effect, but depression tests conducted before and after the snoezelen therapy showed no differences. The authors explained this result as indicating stated that a longer therapeutic period might be required, and they suggested a need to study the effects of long-term therapy on depression. In the present study, we investigated the effects of long-term snoezelen therapy on the emotional states of a stroke patients.

\section{Subjects and Methods}

This study was conducted with one patient in the Korean Veterans Nursing Home who was diagnosed with right hemiplegia due to stroke. The subject was a male Vietnam War veteran, age 80 (Case I, diagnosed in 2008 as left hemiplegia), 81 (Case II, diagnosed in 2005 as right hemiplegia), 78 (Case III, diagnosed in 2009 as right hemiplegia) years, which was suspected to be an after effect of the defoliant exposure. Following his stroke, the subject was hospitalized in the Korean Veterans Hospital and later continued physical therapy as an outpatient. They measured MMSE-K mean score was 24.33 points, indicating that his cognitive function was not damaged. The subject understood the content of the study and positively consented to participate. The study was started with the initial test six weeks after the subject had entered the nursing home, which is the period generally considered as sufficient for a patient to adapt to a nursing home environment. The snoezelen therapy was applied to the subject for four weeks, three times per week, using snoezelen room established in G nursing home. The therapy was applied for 60 minutes each time, with one-onone guidance from a therapist. The stimulations that the patient preferred were provided, and relaxation and sedation were induced. The tools used in the snoezelen therapy included a water pillar, far-infrared radiation, a projector, a model fishbowl, rotating lights and optical fiber pillars for visual stimulation, the sound of water in the water pillar, and music from a stereo for auditory stimulation, a soft chair, tactual tools, a vibrating water bed, and a cushion for tactual stimulation, and aroma for olfactory stimulation.

A reversal $A B A B$ design was applied in order to verify the effect of the snoezelen therapy on the emotional status of the single stroke patient. In all the A-B-A'-B' procedures used in the study, 30 minutes of physical therapy and 20 minutes of occupational therapy were conducted, followed by $60 \mathrm{~min}$ of snoezelen therapy. The profile of mood state (POMS) was employed as the inventory to for measuring the emotional state by snoezelen therapy. For objective measurement, three social workers with at least five years experience, who did not participate directly in the study, conducted the measurements and the mean values of the measurements were used for the analysis.

The POMS was developed for quick and convenient investigation of the affective state that is momentary and easily changed [9]. It consists of a total of 65 questions, including six sub-scales, and evaluates mood state at five levels from 0 to 4 points. The six sub-scales were: tension-anxiety (T) with 9 questions, depression-dejection (D) with 15 questions, anger-hostility (A), vigor-activity (V) with 8 questions, fatigue-inertia (F) with 7 questions, and confusion-bewilderment (C) with 7 questions. Among the 65 questions, 7 questions that did not pertain to any of the abovementioned sub-scales were not graded.

\section{Results}

This study was conducted in a reversal design to investigate the continued effects of snoezelen therapy on the emotional states of stroke patients. The change in the emotional state of this subject, a stroke patient, was measured before and after the snoezelen therapy application. The required time for completion of the pegboard test, performed to examine the physical changes in his psychologically stable state, decreased from 159.78 to 147.28 in the first test and from 160.72 to 141.09 in the second test. The results of the POMS: the score in the T (tension-anxiety) sub-scale with 9 questions was reduced from 13 points to 2 points in the first test and from 10 points to 5 points in the second test; the score in the $\mathrm{D}$ (depression-dejection) sub-scale with 15 questions was 
reduced from 38 points to 25 points in the first test and from 13 points to 12 points in the second test the score in the A (anger-hostility) sub-scale with 12 questions was reduced from 12 points to 9 points in the first test and from 4 points to 0 points in the second test the score in the $\mathrm{V}$ (vigor-activity) sub-scale with 8 questions was increased from 0 points to 3 points in the first test and from 2 points to 6 points in the second test the score in the $\mathrm{F}$ (fatigue-inertia) sub-scale with 7 questions was reduced from 19 points to 4 points in the first test, but increased from 11 points to 14 points in the second test and the score in the $\mathrm{C}$ (confusion-bewilderment) subscale with 7 questions was reduced from 18 points to 7 points in the first test and from 14 points to 5 points in the second test (Table 1). The result of the pegboard test, decreased from 266.47 to 165.78 in the first test and from 137.94 to 129.10 in the second test. And the detailed changes in the scores for the individual questions are as follows: the score in the $\mathrm{T}$ sub-scale was reduced from 13 points to 10 points in the first test and from 10 points to 8 points in the second test; the score in the D sub-scale was reduced from 33 points to 11 points in the first test but increased from 11 points to 14 points in the second test; the score in the A sub-scale was reduced from 9 points to 5 points in the first test and from 5 points to 3 points in the second test; the score in the $\mathrm{V}$ sub-scale was reduced from 25 points to 20 points in the first test but increased from 21 points to 31 points in the second test; the score in the $\mathrm{F}$ sub-scale was increased from 5 points to 8 points in the first test and from 6 points to 12 points in the second test and the score in the C sub-scale was increased from 13 points to 18 points in the first test and from 7 points to 9 points in the second test (Table 2). Case III of result, decreased from 124.47 to 123.41 in the first test and from 147.15 to 134.41 in the second test. The score in the $\mathrm{T}$ sub-scale was reduced from 2 points

Table 1. Age: 80, diagnosed in 2008 as left hemiplegia.

\begin{tabular}{ccccc}
\hline & A & B & A' & B' \\
\hline Assessment Date & 21 Jun & 16 Jul & 16 Aug & 08 Oct \\
Pegboard (sec) & 159.78 & 147.28 & 160.72 & 141.09 \\
Profile Of Mood States Unit: Point & & \\
Tension-Anxiety & 13 & 2 & 10 & 5 \\
Depression-Dejection & 38 & 25 & 13 & 12 \\
Anger-Hostility & 12 & 9 & 4 & 0 \\
Vigor-Activity & 0 & 3 & 2 & 6 \\
Fatigue-Inertia & 19 & 4 & 11 & 14 \\
Confusion-Bewilderment & 18 & 7 & 14 & 5 \\
\hline
\end{tabular}

to 1 points in the first test and from 2 points to 1 points in the second test; the score in the $\mathrm{D}$ sub-scale was reduced from 7 points to 3 points in the first test and from 7 points to 2 points in the second test; the score in the $\mathrm{A}$ sub-scale was reduced from 0 points to 0 points in the first test and from 0 points to 0 points in the second test; the score in the $\mathrm{V}$ sub-scale was increased from 15 points to 19 points in the first test and from 15 points to 18 points in the second test; the score in the F sub-scale was reduced from 10 points to 0 points in the first test and from 3 points to 0 points in the second test; the score in the $\mathrm{C}$ sub-scale was increased from 3 points to 4 points in the first test, but reduced from 5 points to 4 points in the second test (Table 3).

\section{Discussion}

Snoezelen is reported to enhance the cognitive and social functions of dementia patients and mentally retarded children. This is due to its common therapeutic effects. Such as the promotion of positive emotional and mood

Table 2. Age: 81, diagnosed in 2005 as right hemiplegia.

\begin{tabular}{ccccc}
\hline & A & B & A' & B' \\
\hline Assessment Date & 20 Feb & 19 Mar & 16 Apr & 10 May \\
Pegboard (sec) & 266.47 & 165.78 & 137.94 & 129.10 \\
Profile of Mood States Unit: Point & & \\
Tension-Anxiety & 13 & 10 & 10 & 8 \\
Depression-Dejection & 33 & 11 & 11 & 14 \\
Anger-Hostility & 9 & 5 & 5 & 3 \\
Vigor-Activity & 25 & 20 & 21 & 31 \\
Fatigue-Inertia & 5 & 8 & 6 & 12 \\
Confusion-Bewilderment & 13 & 18 & 7 & 9 \\
\hline
\end{tabular}

Table 3. Age: 78, diagnosed in 2009 as right hemiplegia.

\begin{tabular}{ccccc}
\hline & A & B & A' & B' \\
\hline Assessment Date & 20 Feb & 19 Mar & 16 Apr & 10 May \\
Pegboard (sec) & 124.47 & 123.41 & 147.15 & 134.41 \\
Profile of Mood States Unit: Point & & \\
Tension-Anxiety & 2 & 1 & 2 & 1 \\
Depression-Dejection & 7 & 3 & 7 & 2 \\
Anger-Hostility & 0 & 0 & 0 & 0 \\
Vigor-Activity & 15 & 19 & 15 & 18 \\
Fatigue-Inertia & 10 & 0 & 3 & 0 \\
Confusion-Bewilderment & 3 & 4 & 5 & 4 \\
\hline
\end{tabular}


states, which studies have shown include relaxation, peaceful emotions, increased happy feelings, reduced anxiety, decreased hostility, sedative effects, and the alleviation of psychological stress $[7,10]$. Initially developed as a form of leisure and recreation, snoezelen has been provided to those whose intellectual level is low and who are unable to find needed stimulation by themselves. Notwithstanding, it is an intervention method that uses multisensory stimulation and environment to provide a variety of people with therapy for the purpose of reducing negative or antisocial behaviors, increasing sociality and interest in the external world, and improving personal hygienic habits [11]. In the present study, which used an A-B-A'-B' research design, the first test showed that snoezelen reduced tension, anxiety, anger, and depression, while the second test showed similar effects, in addition to fatigue. The application of snoezelen had a positive effect on the emotional stability of a patient with stroke caused by defoliant exposure during the Vietnam War. This result was consistent with the results of previous studies conducted with children who had sustained brain injury, elderly dementia patients, children with mental retardation and learning problems, and chronic pain patients $[8,12]$. The contradictory scores seen for fatigue between the first and second tests (i.e., a decrease in the first test vs. an increase in the second test) may be due to the fact that snoezelen therapy was performed after 30 minutes of physical therapy and 20 minutes of occupational therapy. This should be taken into account in future studies.

The multisensory snoezelen environment helped to recover brain activity and reduce heart rate, muscular tension, and excitation, even though neither ROM and stretching exercise for relaxation were applied nor was the generally applied task-oriented therapeutic approach [13]. Various sensory stimuli improve the synapses conveying nerve stimulations, the reticular activating system, and brain synapse and functions [8]. In this respect, snoezelen is assumed to aid emotional stabilization in stroke patients through varied sensory stimulation.

In the present study, the pegboard implementation test was performed to investigate improvements in attention and cognition following the emotional changes induced by snoezelen application. The stroke patient in the present study showed a continued improvement from the initial measurement to the second test, which indicated that attention and cognition were improved by emotional relaxation.

In summary, snoezelen, which was reported in previous studies to have a positive effect on emotional stability and cognition in patients with emotional instability, may also positively affect emotional stability and cognition in patients with emotional instability due to combat in wars such as the Vietnam War, or with stroke due to defoliant exposure. Activities using sensory instruments in snoezelen are engaged in various ways in accordance with the objectives of the subjects. One principle of using sensory instruments is to use a single stimulation at the outset and slowly add other sensory instruments provided the subject can endure the simulation. Too, mush stimulation can sometimes have negative effects, such as intimidating the subjects or making them nervous [14]. Therefore, sensory stimulations in a snoezelen environment must not all be introduced at the same time, but must be planned in line with the purpose of the intervention and the reactions of the individuals.

\section{REFERENCES}

[1] L. Hardell, M. Eriksson and O. Axelson, "Agent Orange in War Medicine: An Aftermath Myth,” International Journal of Health Services, Vol. 28, No. 4, 1998, pp. 715724. doi:10.2190/L940-B8FK-3Y5E-RG86

[2] N. Brooks and W. Mckinlay, "Return to Work with the First Seven Years after Head Injury,” Brain Injury, Vol. 1, No. 1, 1986, pp. 5-9. doi:10.3109/02699058709034439

[3] K. W. Hope, "The Effects of Multi-Sensory Environments on Older People with Dementia," Journal of Psychiatric and Mental Health Nursing, Vol. 5, No. 5, 1998, pp. 377385. doi:10.1046/j.1365-2850.1998.00143.x

[4] R. Baker, S. Bell, E. Baker, S. Gibson, J. Holloway and R. Pearce, "A Randomized Controlled Trial of the Effects of Multi-Sensory Stimulation(MSS) for People with Dementia,” British Journal of Clinical Psychology, Vol. 40, No. 1, 2001, pp. 81-96. doi:10.1348/014466501163508

[5] J. Hogg, J. Cavet, L. Lambe and M. Smeddle, “The Use of Snoezelen's as Multisensory Stimulation with People with Intellectual Disability: A Review of the Research,” Research in Developmental Disabilities, Vol. 22, No. 5, 2001, pp. 353-372. doi:10.1016/S0891-4222(01)00077-4

[6] R. Hutchinson and L. Hagger, "The Development and Evaluation of a Snoezelen Leisure Resource for People with Multiple Disability,” Chesterfield, Rompa, 1994, pp. 18-48.

[7] P. Schofield and B. Davis, "Sensory Stimulation (Snoezelen) versus Relaxation: A Potential Strategy for the Management of Chronic Pain,” Disability and Rehabilitation, Vol. 22, No. 15, 2000, pp. 675-682. doi:10.1080/096382800445470

[8] G. A. Hotz, "The Effects of Snoezelen Therapy in Children with Traumatic Brain Injury,” 2005. http//www.WorldWide Snoezelen.com

[9] D. M. McNair, M. Lorr and L. F. Droppleman, "Manual for Profile Mood States Manual,” Educational \& Industrial Testing Service, San Diego, 1992.

[10] R. Baker, Z. Dowling, L. A. Wareing, J. Dawson and J. Assey, "Snoezlen: It's Long-Term and Short-Term Effects on Older People with Dementia," British Journal of Occupational Therapy, Vol. 60, 1997, pp. 213-218. 
[11] A. M. Chitsey, B. K. Haight and M. M. Jones, "Snoezelen: A Multisensory Environmental Intervention," Journal of Gerontological Nursing, Vol. 28, No. 3, 2002, pp. 41-49.

[12] G. A. Hotz, A. Castelblanco and I. M. Lara, "Snoezelen: A Controlled Multi-Sensory Stimulation Therapy for Children Recovering from Severe Brain Injury,” Brain Injury, Vol. 20, No. 8, 2006, pp. 879-888. doi:10.1080/02699050600832635
[13] E. Slevin and A. Mclelland, "Multisensory Environment: Are They Therapeutic? A Single Subject Evaluation of the Clinical Effectiveness of a Multi-Sensory Environment,” Journal of Clinical Nursing, Vol. 8, No. 1, 1999, pp. 48-56. doi:10.1046/j.1365-2702.1999.00211.x

[14] S. Fowler, "Multisensory Rooms and Environments,” Jessica Kingsley Publishers, London, 2008. 